\title{
Erratum
}

\section{Radiative Corrections and the Sliding Singlet Mechanism for Light Doublet Higgs Fields}

N. Dragon, N. Marinescu, M.G. Schmidt

Z. Phys. C - Particles and Fields 21, 383 (1984)

Equation (13) in the paper should read

$$
\begin{aligned}
& \delta m_{H}^{2}-\delta m_{\underline{H}}^{2}=24 e^{4}\left(\Delta m_{A}^{2}\right)^{2} \int_{0}^{1} d x \underline{x}(1-x) \\
& \cdot \int \frac{d^{4} p}{(2 \pi)^{4}} \frac{1}{(p+q)^{2}+m_{H}^{2}} \int \frac{d 4 k}{(2 \pi)^{4}} \\
& \cdot \frac{1}{\left[k^{2}+p^{2} \cdot x(1-x)+m_{A}^{2}\right]^{4}}
\end{aligned}
$$

and (14) should be corrected to

$$
V_{1} \sim\left(\Delta m_{A}^{2}\right)^{2} \int_{0}^{\infty} p^{2} d p^{2}\left[f\left(p^{2}, m_{H}^{2}\right)-f\left(p^{2}, \mu^{2}\right)\right]
$$

$$
\cdot \frac{\partial}{\partial p^{2}}\left\{\frac{1}{p \sqrt{p^{2}+4 m_{A}^{2}}} \ln \frac{\sqrt{p^{2}+4 m_{A}^{2}}+p}{\sqrt{p^{2}+4 m_{A}^{2}}-p}\right\} \text {. }
$$

The rough estimates given after (15) apply for $\mu^{2}=0$ $\left(m_{H}^{2}\right)$. More precise we have $\frac{\partial V}{\partial m_{H}^{2}} \sim \frac{\left(\Delta M^{2}\right)^{2}}{M_{A}^{2}} \ln \frac{m_{H}^{2}}{m_{A}^{2}}$ for $m_{H} / m_{A} \ll 1$ and $\sim \frac{\left(\Delta M^{2}\right)^{2}}{M_{H}^{2}} \log \frac{m_{A}^{2}}{m_{H}^{2}}$ for $m_{H} / m_{A} \gg 1$. However, only the powers of $m_{H}^{2}$ enter the discussion in the first part.

Radiative corrections are irrelevant for $\Delta m_{A}^{2} \ll m_{\text {weak }}^{2}$. Inequality (8) and $\Delta m_{A}^{2} \lesssim m_{A}^{2}$ enforce $\Delta m_{A}^{2} \lesssim m_{\text {weak }}^{2}(\mathrm{mod} / \mathrm{log})$ as a necessary condition for the sliding singlet mechanism. 